\title{
Detection of apoptosis in vivo using antibodies against caspase-induced neo-epitopes
}

\author{
Sabine Jakob ${ }^{\text {a }}$, Nadia Corazza ${ }^{\mathrm{a}}$, Eva Diamantis ${ }^{\mathrm{b}}$, Andreas Kappeler ${ }^{\mathrm{a}}$, Thomas Brunner ${ }^{\mathrm{a}, *}$ \\ ${ }^{a}$ Division of Immunopathology, Institute of Pathology, University of Bern, Murtenstrasse 31, 3010 Bern, Switzerland \\ ${ }^{\mathrm{b}}$ General Clinical Histopathology, Institute of Pathology, University of Bern, Murtenstrasse 31, 3010 Bern, Switzerland
}

\begin{abstract}
Cell death induction by apoptosis is an important process in the maintenance of tissue homeostasis as well as tissue destruction during various pathological processes. Consequently, detection of apoptotic cells in situ represents an important technique to assess the extent and impact of cell death in the respective tissue. While scoring of apoptosis by histological assessment of apoptotic cells is still a widely used method, it is likely biased by sensitivity problems and observed-based variations. The availability of caspase-mediated neo-epitopespecific antibodies offers new tools for the detection of apoptosis in situ. Here, we discuss the use of immunohistochemical detection of cleaved caspase 3 and lamin A for the assessment of apoptotic cells in paraffin-embedded liver tissue. Furthermore, we evaluate the effect of tissue pretreatment and antigen retrieval on the sensitivity of apoptosis detection, background staining and maintenance of tissue morphology.
\end{abstract}

Keywords: Apoptosis; Liver; Caspase; Lamin A; Fas/CD95; Immunohistochemistry; Paraffin sections; Histology; Antigen retrieval

\section{Introduction}

Cell death has been recognized as essential mechanism to counter-balance cell division and proliferation in order to maintain tissue homeostasis. Aberrant excessive cell death has been implicated in various forms of disease-associated tissue damage whereas reduced cell death is a typical feature of cancerous tissues [1,2]. Apoptosis represents a defined form of a tightly controlled, programmed cell death, distinct from the accidental death occurring during necrosis. It was been originally described as a specific and morphologically distinct form of cell death in various tissues $[3,4]$. Consequently, the morphological assessment of apoptotic cells by light or electron microscopy has been for a long time one of the gold standards of in situ apoptosis detection and is still frequently used to describe apoptotic cell death in tissues. However, scoring of apoptosis on

\footnotetext{
* Corresponding author. Fax: +41313818764.

E-mail address: tbrunner@pathology.unibe.ch (T. Brunner).
}

tissue sections using routine histological stainings is tedious and observer-dependent $[3,5]$. In addition, characterization of the different biochemical processes, leading to the distinct morphological changes of apoptotic cells, i.e. cellular and nuclear condensation and fragmentation, has pointed out that these morphological changes occur relatively late after cell death induction. Furthermore, apoptotic cells display relatively early on different eat-me signals, resulting in rapid phagocytosis and the removal of apoptotic cells [6]. Consequently, a scoring of apoptotic cell death in tissue sections solely based on morphological changes might largely underestimate the real frequency of dying cells. A classical example for the inefficient detection of apoptosis by morphological techniques, respectively efficient removal of apoptotic cells, is the thymus where approximately $95 \%$ of all thymocytes die by apoptosis, yet only a minor fraction is observed as displaying apoptotic morphologies [7-9].

This clearly demonstrates the need of more sensitive methods to detect apoptotic cells in tissue sections. Such 
enhanced morphology-based detection systems are not only important for in vivo apoptosis studies in basic research, but represent also important diagnostic tools for pathologists and disease diagnosis. Not surprisingly, reduced or increased apoptotic cell death in tissue specimens has been associated with various diseases and correlations with severity of disease and/or patients survival have been demonstrated [10-12].

Early on in the characterization of apoptosis DNA fragmentation has been recognized as one of the hallmarks of apoptosis [9]. Since its introduction [13,14] the TUNEL (terminal deoxynucleotide transferase-mediated dUTP-biotin nick-end labeling) assay has been extensively used for the detection of apoptosis-associated DNA strand breaks in cultured cells and tissue sections. While the TUNEL technique has certain advantages, e.g. that it can be combined with immunohistochemistry or other antibody-based techniques [15], it has also various disadvantages. A major problem is the fact that DNA fragmentation is not restricted to apoptosis but also occurs in necrotic cells, only the frequency of DNA strand breaks is increased in apoptotic cells. Similarly, overfixation of tissue samples in formalin may cause DNA breaks, which are further exposed after pretreatment of tissue sections with proteinase $\mathrm{K}$ to allow the terminal deoxynucleotide transferase get access to the nuclei. Finally, the extent and duration of proteinase $\mathrm{K}$ digestion largely defines the sensitivity and the background of this technique. Thus, TUNEL is considered a technically demanding apoptosis detection method for experienced users, requiring a large panel of positive and negative controls $[5,16]$.

The aspartate-specific cysteine proteases, caspases, are key effector molecules of apoptotic cell death [17-19]. Caspases are expressed in most cells and activated upon most apoptotic stimuli. Importantly, the typical morphologic changes occurring in apoptotic cells largely depend on caspase activity. Current estimates propose up to 400 different caspase substrates, which become proteolytically cleaved during apoptosis, and cleavage of some substrates has been correlated with specific morphological changes occurring in apoptotic cells [20,21]. Caspase activation and substrate cleavage can be easily monitored in vitro by detecting their processing using Western blots and specific antibodies [22]. These techniques are however not applicable to in situ apoptosis detection on tissue sections. The discovery that caspases and caspase substrates are cleaved at very specific sites leading to neo-epitopes with defined amino acid sequences has led to the generation of antibodies reacting only with cleaved (activated) caspases and caspase substrate $[23,24]$. As the generation of these neo-epitopes is dependent on apoptosis induction and caspase activation, such antibodies represent useful and valuable tools for detecting apoptosis-associated processes in cell culture and on tissues sections [5].

Currently, numerous antibodies against various cleaved (active) caspases or cleaved caspase substrates are commercially available and have been used for apoptosis detection in situ [23-25]. However, not all caspases or caspase substrates may be equally well expressed and/or cleaved in different cell types or after apoptosis induction by different triggers. Caspase 3 is a critical effector caspase, expressed in most cells and activated in response to most apoptosis triggers [22]. Similarly, lamin A is a ubiquitously expressed nuclear protein, which is cleaved in a caspase-dependent manner during apoptosis [26,27]. In this study we describe the use of anti-cleaved caspase 3 and anti-cleaved lamin A for the detection of apoptosis in formalin-fixed mouse liver tissue using immunohistochemistry. Specifically, we address the role of tissue pretreatments and antigen retrieval on the sensitivity of apoptosis detection and maintenance of tissue morphology. We exclusively focused on paraffin-embedded tissue as the histology is preserved much better than in cryosections and paraffin-embedded tissue is often more readily available, e.g. in tissue banks and diagnostic archives.

\section{Materials and methods}

\subsection{Induction of apoptosis in mouse liver tissue}

Apoptosis induction in murine liver tissue was performed as described previously by Nagata and colleagues [28], and modified by Corazza et al. [29]. The injection of the agonistic anti-Fas (CD95) antibody (clone Jo-2) leads to rapid stimulation of the Fas receptor and induction of massive hepatocyte apoptosis. Female $\mathrm{C} 57 \mathrm{Bl} / 6$ mice (7-10 weeks) were weighted to calculate the antibody/body weight ratio, and immobilized in a mouse strainer. AntiFas antibody $(0.25 \mu \mathrm{g} / \mathrm{g}$ body weight, clone Jo-2, e-bioscience, San Diego, CA) or PBS control was injected i.v. into the tail vein using a $1 \mathrm{ml}$ tuberculin syringe and a $27 \mathrm{G}$ needle. Control animals received i.v. injection of PBS only. After $4 \mathrm{~h}$ mice were euthanized with a $\mathrm{CO}_{2}$ overdose, liver samples were immediately resected and fixed overnight in 4\% paraformaldehyde (Sigma) in PBS. Fixed liver tissue was then dehydrated in an increasing ethanol row and embedded in paraffin using a Tissue Tek II Tissue Embedding Center (Haska, Bern, Switzerland). Five um sections were then cut on a Jung SM 200R microtome (Leica, Nussloch, Germany) and mounted on microscope glass slides (Superfrost Plus, Thermo Fisher Scientific). Sections were dried for $2 \mathrm{~h}$ at $57^{\circ} \mathrm{C}$, and deparafinized and rehydrated for $2 \times 10 \mathrm{~min}$ in xylole, $5 \mathrm{~min}$ in $100 \%$ ethanol, and then for $20 \mathrm{~s}$ each in $95 \%, 95 \%, 70 \%, 70 \%, 35 \%$ ethanol, $\mathrm{H}_{2} \mathrm{O}$ and $25 \mathrm{mM}$ TBS $\mathrm{pH} 7.5$.

\subsection{Pretreatments (antigen retrieval)}

Fixation of the tissue leads to a variable degree to the denaturation and crosslinking of the antigen of interest with other proteins. This leads to antigen masking and the inability of the antibody to recognize the antigen. As a consequence various tissue pretreatment methods have been developed that lead to the retrieval of the antigen. 
Certain pretreatments may however only work for certain antigens and with certain antibodies, and therefore various pretreatments have to be tested for individual antibodies. Another important consideration is the fact that pretreatments variably affect the tissue morphology and thus the identification of distinct cell types and tissue structures. Here we describe a series of pretreatments typically used for immunohistochemical detection of antigen on paraffin sections.

\subsubsection{Microwave-citrate ( $M W$-citrate)}

Tissue sections were placed in a glass container with $220 \mathrm{ml} 10 \mathrm{mM}$ citrate buffer $\mathrm{pH}$ 6.0, containing approx. 6 boiling chips. The container was covered and tissues were treated for $8 \mathrm{~min}$ with $850 \mathrm{~W}$, followed by $2 \times 5 \mathrm{~min}$ with $410 \mathrm{~W}$ in a microwave with a rotary plate. Sections were then cooled down for $15 \mathrm{~min}$ in the same buffer removing the container lid. Before staining slides were quickly rinsed in $25 \mathrm{mM}$ Tris $\mathrm{pH} 7.5$.

\subsubsection{Microwave-urea ( $M W$-urea)}

Tissue sections were placed in a glass container with $220 \mathrm{ml} \mathrm{5 \%}$ urea in $100 \mathrm{mM}$ Tris $\mathrm{pH}$ 9.5, containing approx. 6 boiling chips. Sections were then microwave treated as described above under Section 2.2.1.

\subsubsection{Microwave-EDTA ( $M W$-EDTA)}

Tissue sections were placed in a glass container with $220 \mathrm{ml} 1 \mathrm{mM}$ EDTA in $10 \mathrm{mM}$ Tris $\mathrm{pH} 9.0$, containing approx. 6 boiling chips. Sections were then microwave treated as described above under Section 2.2.1.

\subsubsection{Steam}

Tissue sections were placed in a metal slide rack. A volume of 1.51 of $10 \mathrm{mM}$ citrate buffer $\mathrm{pH} 6.0$ was heated to the boiling point in a pressure cooker with open lid. Tissue sections were placed in the boiling buffer and the lid is immediately closed. Heat was reduced and time was stopped. After 5 min pressure cooker was cooled with cold running water, the lid was opened and sections were immediately placed in cold $\mathrm{H}_{2} \mathrm{O}$.

\subsubsection{Steam $10 \mathrm{~min}$}

Tissue sections were treated as described above under Section 2.2.4, but heated for $10 \mathrm{~min}$ in the pressure cooker.

\subsubsection{Trypsin}

Tissue sections were digested for $20 \mathrm{~min}$ at $37^{\circ} \mathrm{C}$ in $0.1 \%$ trypsin (Difco) in $25 \mathrm{mM}$ Tris pH 8.0, $140 \mathrm{mM} \mathrm{NaCl}, 10 \mathrm{mM}$ $\mathrm{CaCl}_{2}$ in a glass cuvette placed in a water bath. The solution without trypsin was prewarmed at $37^{\circ} \mathrm{C}$, trypsin was added just before sections were placed into the solution. Tissue sections were then washed in $25 \mathrm{mM}$ Tris $\mathrm{pH}$ 7.5.

\subsubsection{Pronase}

Glass slides with tissue sections were dried at the lower side using paper towels. Sections were then placed on a heating plate $\left(40{ }^{\circ} \mathrm{C}\right)$ and immediately covered with 70 $100 \mu \mathrm{l}$ pronase solution $(0.1 \%$ pronase from St. griseus, Roche, in $50 \mathrm{mM}$ TBS pH 7.5). After 6 min sections were washed in $25 \mathrm{mM}$ Tris $\mathrm{pH} 7.5$.

\subsection{Immunohistochemistry}

After pretreatments tissue sections were quickly rinsed in TBS pH 7.4 and then incubated for 10 min at RT with endogenous peroxidase blocking solution (DakoCytomation, Ready-to-use, S2001). After that unspecific binding was blocked by incubating slides with $5 \%$ goat serum, $0.5 \%$ casein in TBS pH 7.4 (antibody dilution buffer) for $10 \mathrm{~min}$. The tissue was then stained with the primary antibody diluted in antibody dilution buffer for $60 \mathrm{~min}$ at RT. Both, rabbit anti-cleaved caspase 3 (Cell Signaling Technology, 5A1) and rabbit anti-cleaved lamin A (Cell Signaling Technology, 2035) were diluted 1:100. As staining control purified rabbit immunoglobulin (Sigma) was used at $1 \mu \mathrm{g} / \mathrm{ml}$. Sections are then briefly dipped in TBS, and washed $2 \times 5 \mathrm{~min}$ in TBS. The secondary biotinylated goat anti-rabbit antibody (DakoCytomation) was diluted 1:100 in antibody dilution buffer and sections were stained for $45 \mathrm{~min}$ at RT. Sections were washed $2 \times 5 \mathrm{~min}$ in TBS prior to incubation with the pre-aggregated avidin-biotin $(\mathrm{AB})$ complex (DakoCytomation). The AB complex was prepared by diluting reagents $\mathrm{A}$ (avidin) and $\mathrm{B}$ (biotinylated horse radish peroxidase) 1:200 in TBS. At least 30 min were allowed to form complexes. Sections were covered with $100 \mu 1$ of the $\mathrm{AB}$ complex and incubated for $45 \mathrm{~min}$ at RT. After $2 \times 5$ min washing in TBS, immunohistochemistry was developed by incubating sections for 7-8 $\mathrm{min}$ in the dark in horse radish peroxidase substrate solution $(0.2 \mathrm{mg} /$ $\mathrm{ml}$ 3,3'-diaminobenzidine tetrahydrochloride DAB, $0.012 \%$ $\mathrm{H}_{2} \mathrm{O}_{2}, 20 \mathrm{mM}$ citric acid monohydrate, $100 \mathrm{mM}$ imidazole, $100 \mathrm{mM} \mathrm{NaCl}, \mathrm{pH}$ 7.0) in a glass cuvette. Slides were washed $2 \times 5 \mathrm{~min}$ in TBS and counterstained for $1 \mathrm{~min}$ with hematoxilin, followed by rinsing in tab water. Sections were then dehydrated $(2 \times 20$ s $35 \%$ ethanol, $2 \times 20$ s $70 \%$ ethanol, $2 \times 20 \mathrm{~s} 95 \%$ ethanol, $2 \times 2 \mathrm{~min} 100 \%$ ethanol, $2 \times 2$ min xylole, 5 min xylole) and mounted with Eukitt mounting medium (Electron Microscopy Sciences) and appropriate cover slips.

\section{Results and discussion}

\subsection{Fas-induced liver cell apoptosis}

The aim of this study was to demonstrate and discuss the effects of different tissue pretreatment on the sensitivity and background of apoptosis detection in situ using anticleaved caspase 3 and anti-cleaved lamin A antibodies. As a study tissue we chose liver tissue from mice previously injected with agonistic anti-Fas antibody. Murine hepatocytes express high levels of Fas receptor and are exquisitely sensitive to the pro-apoptotic activity of Fas receptor-activating triggers, including agonistic anti-Fas antibody, 
recombinant FasL and FasL-expressing activated $\mathrm{T}$ cells and NK cells [28-31]. Importantly, apoptosis induction via Fas is fast (induced within few hours), but the extent is dependent on the concentration of agonist. This allows the induction and detection of various degrees of apoptosis and tissue damage, ranging from few dispersed cells to massive apoptosis affecting large areas of the liver. In this study we employed a relative low amount of anti-Fas antibody and consequently induced only minimal liver damage in order to more easily distinguish between positive, negative and false positive cells. Critically, anti-Fas-induced apoptosis is completely dependent on caspase activity and caspase inhibition by protease inhibitors strongly inhibits anti-Fas-induced liver damage [32]. Fas-induced apoptosis is also representative model for immunopathology-mediated apoptosis in the liver during various forms of hepatitis, as this also largely depends on Fas ligand-Fas interaction $[30,33,34]$. In addition, liver tissue is a frequent target of apoptosis-inducing side effects of drugs, for example chemotherapy and paracetamol overdose [35,36].

Another aspect makes the liver an interesting model to study tissue pretreatment protocols on apoptosis detection by immunohistochemistry. Many immunohistochemistry detection and visualization systems depend on avidin-biotin-mediated amplification of the signal. The liver is, however, a rich source of endogenous biotin and different tissue pretreatments differentially affect background staining by permitting access of endogenous biotin to the visualization system. In particular harsh tissue pretreatment protocols, such as using urea and EDTA, often lead to a uniform background staining of liver tissue by avidin-biotin-based visualization systems (Fig. 1E and F).

\subsection{Detection of cleaved caspase 3}

For this study we employed the anti-cleaved caspase 3 antibody from Cell Signaling Technology [29], however, antibodies from other companies may work equally well and have been used to detect active caspase 3 in tissue sections [23]. Monoclonal antibodies, including the monoclonal rabbit anti-cleaved caspase 3 antibody used (clone 5A1), have the advantage that exact concentrations are known and accurate isotype controls can be used to test for unspecific background staining. Our study demonstrates that the different tissue pretreatments also differently affect background staining. Whereas in general very few false positive cells were observed after staining with the isotype control antibody, pretreatment with EDTA and Urea led to a uniform and intense background staining due to detection of endogenous biotin in the hepatocytes (Fig. 1E and F).

Staining of the tissue sections with anti-cleaved caspase 3 revealed that in the absence of pretreatment no positive staining could be detected, even if apoptotic cells could be identified by morphology (Fig. 1I). Thus, different tissue pretreatments and antigen retrieval methods were tested. Pretreatment with microwave or steam for 5 or $10 \mathrm{~min}$ led to numerous positive cells, some of them with clear apoptotic nuclear morphology, e.g. condensed DNA or fragmented nuclei, whereas others appeared normal and likely represented cells at an early stage of apoptosis (Fig. 1J-L, and Fig. 2A-D). Overall, pretreatment with steam (either 5 or $10 \mathrm{~min}$ ) led to stronger signals and positive cells were more easily detectable than after microwave treatment. However, in particular pretreatment with steam for $10 \mathrm{~min}$ resulted in a significantly reduced tissue morphology (Fig. 1D and L).

Although cleaved caspase 3-positive cells could be easily detected in EDTA and urea pretreated liver tissue, the background was uniformly strong providing very little contrast between background and positive signal (Fig. 1M and $\mathrm{N})$. The enzymatic pretreatment with pronase or trypsin resulted in some unspecific staining and no clearly detectable positive signals (Fig. $1 \mathrm{O}$ and $\mathrm{P}$ ).

In summary, we observed that tissue pretreatment is required for apoptosis detection using anti-active caspase 3 , and that pretreatment with microwave or steam resulted in the best compromise between maintenance of tissue morphology and sensitivity of the immunohistochemistry (Fig. 1J and $\mathrm{K}$ ). As not all cells undergoing apoptosis have equal levels of cleaved caspase 3, the sensitivity of the immunohistochemistry is important for the sensitive detection of apoptotic cells. On the other hand, the preservation of tissue morphology further helps to score apoptotic cells, as the apoptotic morphology together with positive immunohistochemistry signals helps to better spot and score apoptotic cells.

\subsection{Detection of cleaved lamin A}

As with cleaved caspase 3, cleaved lamin A was not detectable by immunohistochemistry in untreated paraformaldehyde-fixed liver sections (Fig. 1Q). In marked contrast, pretreatment with microwave and steam led to the detection of numerous positive cells with a typical ringshaped staining due to the expression of lamin $\mathrm{A}$ in the nuclear membrane (Fig. 1R-T). Similar to cleaved caspase 3 steam pretreatment resulted in a more intense staining, however, steam treatment for $10 \mathrm{~min}$ also reduced the tissue morphology (Fig. 1S and T). While most positive cells displayed this typical nuclear membrane staining, different intensities and localizations were also observed (Fig. 2E$\mathrm{H})$. While in some cells the nuclear membrane staining was barely detectable (Fig. 2E), in some cells it was very intense, dispersed throughout the nucleus (Fig. 2F) and often paralleled with DNA condensation (Fig. 2G). In late apoptotic cells a positive staining was even observed in the cytoplasm (Fig. 2H). This is in agreement with the finding that lamin A fragments can translocate to the cytoplasm [37].

EDTA and urea pretreatment resulted in the sensitive detection of positive cells, however, the high background staining made a scoring of apoptotic cells more difficult (Fig. 1U and V). Enzymatic pretreatment with pronase 

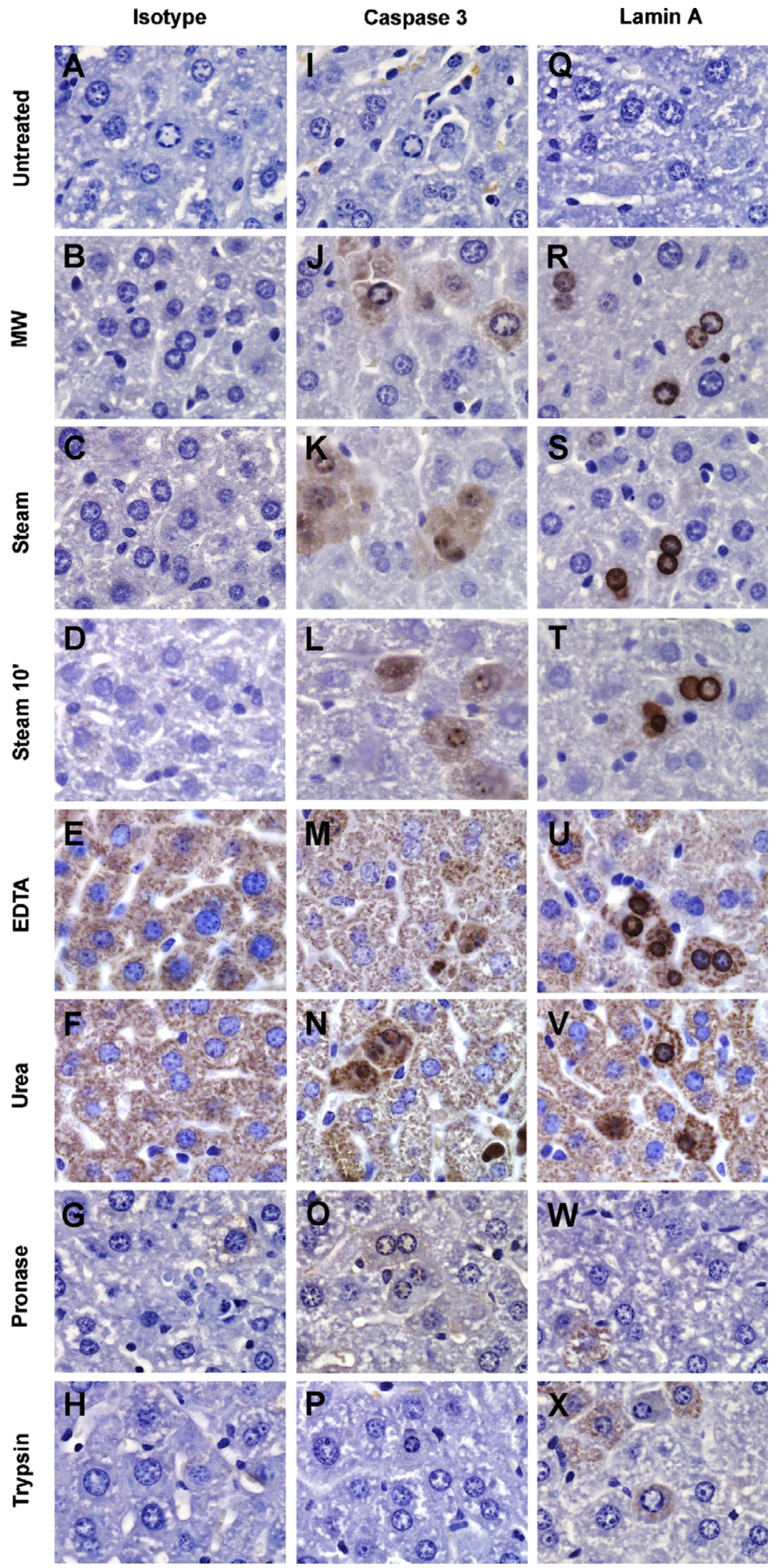

Fig. 1. Effects of tissue pretreatment on immunohistochemical detection of cleaved caspase 3 and lamin A. Liver sections from a mouse injected with agonistic anti-Fas antibody were pretreated as indicated on the left: untreated MW (microwave-citrate buffer), steam (steam 5 min), steam $10^{\prime}$ (steam $10 \mathrm{~min}$ ), EDTA (microwave-EDTA), urea (microwave-urea), pronase, trypsin. Sections were then stained with isotype control (A-H), anti-cleaved caspase 3 (caspase 3, I-P) or anti-cleaved lamin A (lamin A, Q-X). Positive staining was visualized with horse radish peroxidase/DAB (brown staining). 


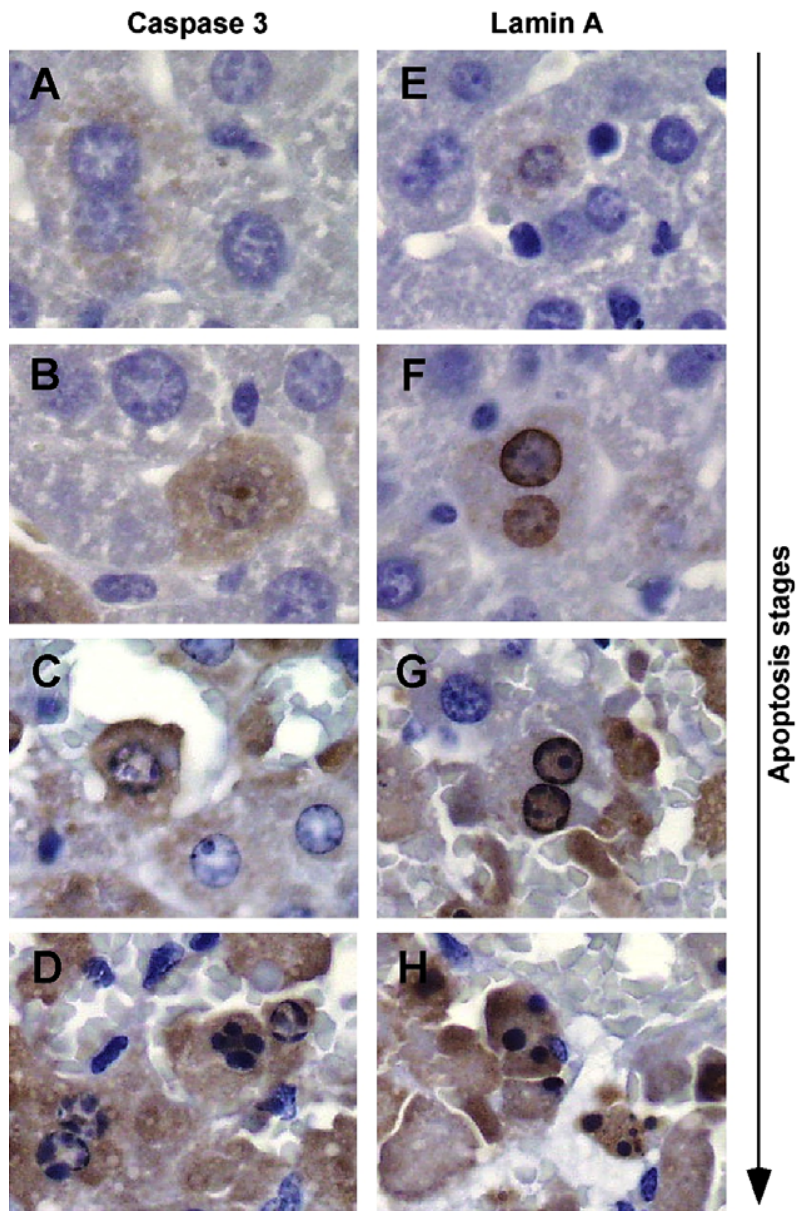

Fig. 2. Detection of cleaved caspase 3 and lamin A at different stages of hepatocyte apoptosis. Liver sections were pretreated with steam $5 \mathrm{~min}$ and stained for cleaved caspase 3 (caspase 3, A-D) and cleaved lamin A (lamin A, E-H). Typical examples of positive cells at different stages of apoptotic cell death are shown. Positive staining is shown in brown.

and trypsin often resulted in false positive cytoplasmic staining and no cleaved lamin A-positive cells with typical nuclear membrane staining (Fig. $1 \mathrm{~W}$ and $\mathrm{X}$ ).

In summary, we observed that pretreatment with steam resulted in the most sensitive detection of cleaved lamin Apositive apoptotic cells with low background staining, while steam pretreatment for $5 \mathrm{~min}$ was advantageous over steam for 10 min due to preservation of the tissue morphology. As with cleaved caspase 3, immunohistochemistry for cleaved lamin A detected cells at different stages of apoptosis, with typical nuclear membrane staining, and more dispersed nuclear and cytoplasmic staining, going along with the occurrence of apoptotic nuclear morphologies.

\section{Concluding remarks}

The aim of this study was to provide detailed protocols for the pretreatment of paraffin-embedded tissue and the in situ detection of apoptosis using anti-cleaved caspase 3 and lamin A antibodies. Our study clearly revealed that antigen retrieval by tissue pretreatment is a prerequisite for positive staining of apoptotic cells with anti-cleaved caspase 3 or lamin A antibodies. While fixation of tissue specimens with formalin and embedding in paraffin may appear disadvantageous at the first glance due to increased length and steps of the staining procedure, the morphology of paraffin-embedded tissue is superior over that obtained with cryosections. A combination between pretreatment of tissue sections with steam and citrate buffer, and the staining with anti-cleaved caspase 3 or lamin A represents a good compromise between preservation of tissue morphology and sensitive detection of apoptotic cells in situ. Tissue banks and diagnostic archives are also a rich source of paraffin-embedded tissue specimens from patient material, and thereby represent an important tool and source for the analysis of apoptotic cell death in different diseases. The different tissue pretreatment protocols described here may also be employed to optimize staining conditions for other antibodies against apoptosis-related antigens.

The question of whether to use anti-cleaved caspase 3 or lamin A, or any other antibody recognizing caspaseinduced neo-epitopes, is rather a matter of scientific interest and taste. Caspase 3 and lamin A are expressed ubiquitously and are cleaved at substantial levels in cell undergoing apoptosis. Consequently, both antibodies allowed the sensitive detection of apoptotic cells. While the relatively faint cytoplasmic staining of cleaved caspase 3 in early apoptotic cells may be somewhat more difficult to score (Fig. 2A and B), the typical nuclear membrane staining of cleaved lamin A is easily detected and allows sensitive scoring of apoptotic cells at various stages (Fig. 2E-H). The appearance of typical apoptotic morphologies, in combination with cleaved caspase 3- or lamin A-positive cells, further helps to identify apoptotic cells in tissue sections.

In summary, detection of apoptotic cells in tissue sections using anti-cleaved caspase or anti-cleaved caspase substrate antibodies offers a sensitive and easy to handle alternative to cumbersome morphological scoring or TUNEL assay.

\section{Acknowledgments}

This work was supported by the Swiss National Science Foundation, the Crohn's and Colitis Foundation of America and the Swiss Cancer League.

\section{References}

[1] D.R. Green, G.I. Evan, Cancer Cell 1 (2002) 19-30.

[2] T. Brunner, C. Mueller, Essays Biochem. 39 (2003) 119-130.

[3] A.H. Wyllie, J.F. Kerr, A.R. Currie, Int. Rev. Cytol. 68 (1980) 251306.

[4] J.F. Kerr, A.H. Wyllie, A.R. Currie, Br. J. Cancer 26 (1972) 239-257.

[5] C. Stadelmann, H. Lassmann, Cell Tissue Res. 301 (2000) 19-31.

[6] J. Savill, V. Fadok, Nature 407 (2000) 784-788.

[7] C.D. Surh, J. Sprent, Nature 372 (1994) 100-103.

[8] H.M. Seitz, T.D. Camenisch, G. Lemke, H.S. Earp, G.K. Matsushima, J. Immunol. 178 (2007) 5635-5642. 
[9] A.H. Wyllie, Nature 284 (1980) 555-556.

[10] D. Todd, G. Yang, R.W. Brown, J. Cao, V. D'Agati, T.S. Thompson, L.D. Truong, Hum. Pathol. 27 (1996) 1012-1017.

[11] A. Chakravarti, E. Noll, P.M. Black, D.F. Finkelstein, D.M. Finkelstein, N.J. Dyson, J.S. Loeffler, J. Clin. Oncol. 20 (2002) 1063-1068.

[12] M. Arai, A. Sasaki, N. Saito, Y. Nakazato, Pathol. Int. 55 (2005) $122-129$.

[13] S.A. Ben-Sasson, Y. Sherman, Y. Gavrieli, Methods Cell Biol. 46 (1995) 29-39.

[14] Y. Gavrieli, Y. Sherman, S.A. Ben-Sasson, J. Cell Biol. 119 (1992) 493-501.

[15] X. Zhang, T. Brunner, L. Carter, R.W. Dutton, P. Rogers, L. Bradley, T. Sato, J.C. Reed, D. Green, S.L. Swain, J. Exp. Med. 185 (1997) $1837-1849$.

[16] K.R. Jerome, C. Vallan, R. Jaggi, Pathology 32 (2000) 186-190.

[17] N.A. Thornberry, Y. Lazebnik, Science 281 (1998) 1312-1316.

[18] S.J. Martin, D.R. Green, Cell 82 (1995) 349-352.

[19] E.M. Creagh, S.J. Martin, Biochem. Soc. Trans. 29 (2001) 696-702.

[20] U. Fischer, R.U. Janicke, K. Schulze-Osthoff, Cell Death Differ. 10 (2003) 76-100.

[21] A.U. Luthi, S.J. Martin, Cell Death Differ. 14 (2007) 641-650.

[22] E.A. Slee, C. Adrain, S.J. Martin, Cell Death Differ. 6 (1999) 10671074.

[23] A. Srinivasan, K.A. Roth, R.O. Sayers, K.S. Shindler, A.M. Wong, L.C. Fritz, K.J. Tomaselli, Cell Death Differ. 5 (1998) 1004-1016.

[24] L. Rydlander, E. Ziegler, T. Bergman, E. Schoberl, G. Steiner, A.C. Bergman, A. Zetterberg, M. Marberger, P. Bjorklund, T. Skern, R. Einarsson, H. Jornvall, Eur. J. Biochem. 241 (1996) 309-314.
[25] H. Bantel, A. Lugering, C. Poremba, N. Lugering, J. Held, W. Domschke, K. Schulze-Osthoff, Hepatology 34 (2001) 758-767.

[26] A. Takahashi, E.S. Alnemri, Y.A. Lazebnik, T. Fernandes-Alnemri, G. Litwack, R.D. Moir, R.D. Goldman, G.G. Poirier, S.H. Kaufmann, W.C. Earnshaw, Proc. Natl. Acad. Sci. USA 93 (1996) 83958400.

[27] L. Rao, D. Perez, E. White, J. Cell Biol. 135 (1996) 1441-1455.

[28] J. Ogasawara, R. Watanabe-Fukunaga, M. Adachi, A. Matsuzawa, T. Kasugai, Y. Kitamura, N. Itoh, T. Suda, S. Nagata, Nature 364 (1993) 806-809.

[29] N. Corazza, S. Jakob, C. Schaer, S. Frese, A. Keogh, D. Stroka, D. Kassahn, R. Torgler, C. Mueller, P. Schneider, T. Brunner, J. Clin. Invest. 116 (2006) 2493-2499.

[30] T. Kondo, T. Suda, H. Fukuyama, M. Adachi, S. Nagata, Nat. Med. 3 (1997) 409-413.

[31] M.J. Pinkoski, T. Brunner, D.R. Green, T. Lin, Am. J. Physiol. Gastrointest. Liver Physiol. 278 (2000) G354-G366.

[32] I. Rodriguez, K. Matsuura, C. Ody, S. Nagata, P. Vassalli, J. Exp. Med. 184 (1996) 2067-2072.

[33] K. Takeda, Y. Hayakawa, L. Van Kaer, H. Matsuda, H. Yagita, K. Okumura, Proc. Natl. Acad. Sci. USA 97 (2000) 5498-5503.

[34] K. Seino, N. Kayagaki, K. Takeda, K. Fukao, K. Okumura, H. Yagita, Gastroenterology 113 (1997) 1315-1322.

[35] G.E. Kass, Chem. Biol. Interact. 163 (2006) 145-159.

[36] S. Ghavami, M. Hashemi, K. Kadkhoda, S.M. Alavian, G.H. Bay, M. Los, Med. Sci. Monit. 11 (2005) RA337-RA345.

[37] J.L. Broers, N.M. Bronnenberg, H.J. Kuijpers, B. Schutte, C.J. Hutchison, F.C. Ramaekers, Eur. J. Cell Biol. 81 (2002) 677691. 